6

7

8

\title{
Problem-Focused Coping Underlying Lower Caregiver Burden in ALS-FTD: Implications for
} Caregiver Intervention

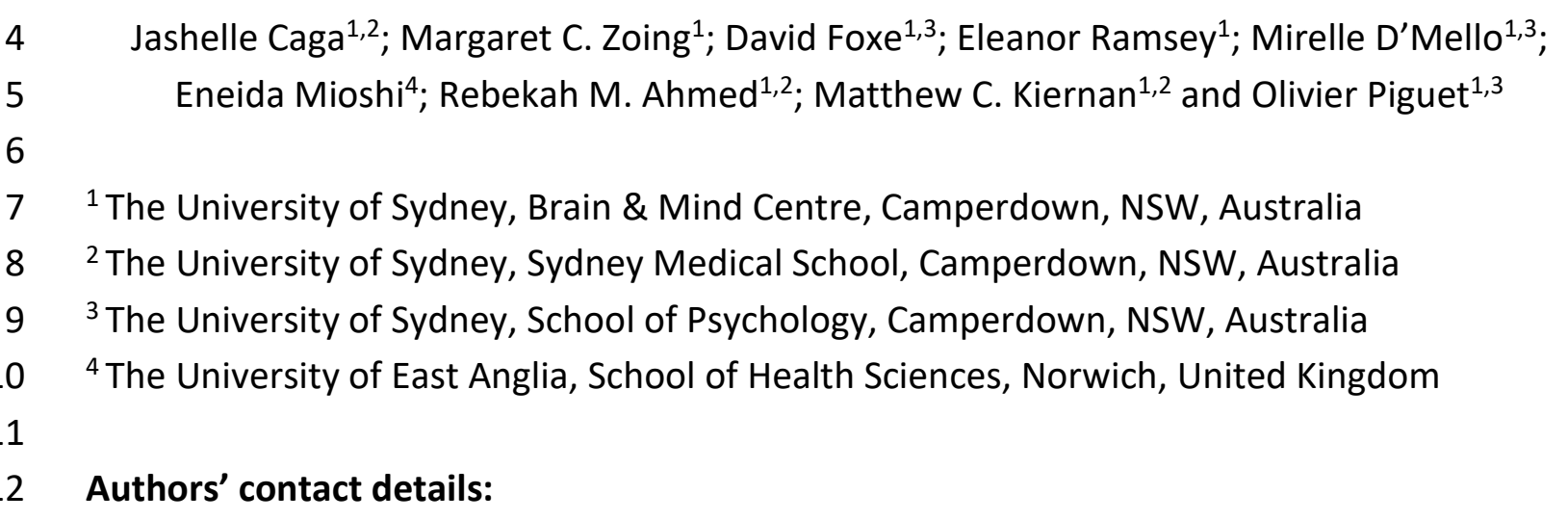

2 Authors' contact details:

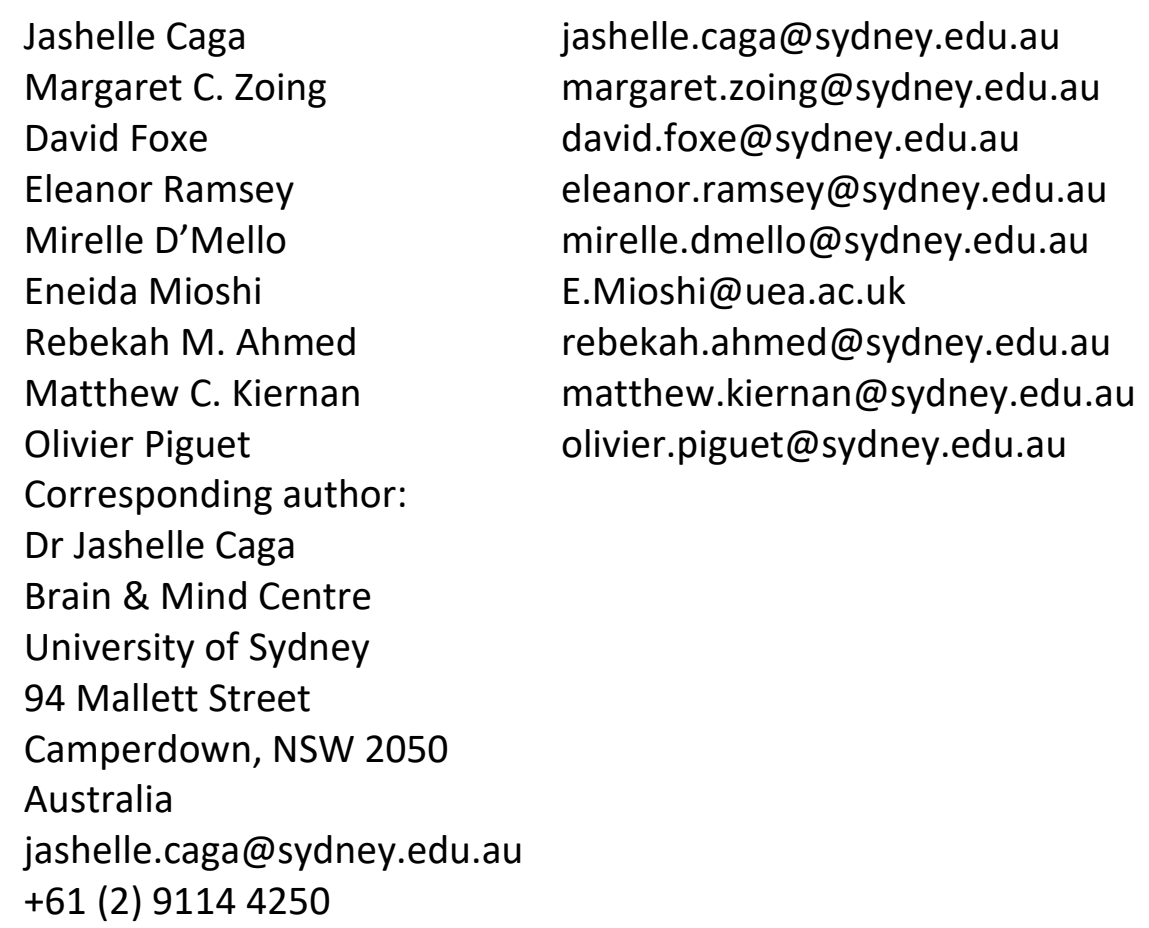

jashelle.caga@sydney.edu.au margaret.zoing@sydney.edu.au david.foxe@sydney.edu.au eleanor.ramsey@sydney.edu.au mirelle.dmello@sydney.edu.au E.Mioshi@uea.ac.uk rebekah.ahmed@sydney.edu.au matthew.kiernan@sydney.edu.au olivier.piguet@sydney.edu.au

14 Word count: 2,918 (excluding abstract, acknowledgments, disclosure statement and references) 
2 Objective: Amyotrophic lateral sclerosis (ALS) is a multisystem neurodegenerative disorder

3 which includes cognitive and behavioural symptoms akin to frontotemporal dementia (FTD).

4 Despite the necessity of caregiver intervention to assist with the management of cognitive

5 and behavioural symptoms, there has been a lack of research on the topic. A focus on

6 caregiver coping may offer a promising foundation to guide the development of

7 interventions as part of ALS care. Accordingly, the aim of the present study was to examine

8 the relationships between caregiver coping, psychological morbidity and burden of care in

9 the context of ALS cognitive and behavioural symptoms.

Methods: Fifty-five patient-caregiver dyads were recruited from specialised ALS and FTD clinics. Specific coping strategies were examined using the COPE Inventory/Brief COPE and psychological morbidity and burden were assessed using the Depression, Anxiety, and Stress Scale-21 and Zarit Burden Interview. The relationship between coping, psychological morbidity and burden of care were analysed using univariate and multivariate methods.

Results: High-burden caregivers were more likely to be caring for patients with a diagnosis of ALS-FTD ( $p=.0001$ ). Caregivers used problem-focused strategies (particularly planning) more frequently $(M=71.4, S D=15.3)$ compared to emotion-focused $(M=60.8, S D=12.3)$ and dysfunctional coping strategies $(M=42.2, S D=8.6)$. A diagnosis of ALS-FTD $(p=.0001)$ and problem-focused strategies ( $p=.024)$ emerged as significant predictors of caregiver burden. Caregiver anxiety, depression and stress were not predictive of caregiver burden $(p=.151)$. patients may mitigate caregiver burden. 
1 Keywords: Amyotrophic lateral sclerosis, frontotemporal dementia, caregivers, coping

2 strategies, and burden.

3

4 
2 Increasing evidence indicates that amyotrophic lateral sclerosis (ALS) and frontotemporal

3 dementia (FTD) represent parts of a clinico-pathological spectrum (1-4). Based on

4 population studies, the incidence of cognitive and behavioural changes in ALS vary between

$510-75 \%$, with up to $14 \%$ of patients meeting the criteria for a diagnosis of concomitant

6 frontotemporal dementia (FTD) $(5,6)$. Conversely, $10-15 \%$ of individuals diagnosed with FTD

7 have concomitant ALS and another $25-30 \%$ will exhibit motor neuron dysfunctions that do

8 not meet diagnostic criteria for $\operatorname{ALS}(7,8)$.

9

Cognitive and behavioural changes inevitably place a heavy burden on ALS caregivers (9-14).

In addition to dealing with patients' rapid physical decline, caregivers must also cope with cognitive and behavioural symptoms that entail additional responsibilities such as supervising, assisting with decision making (14), prompting or taking over tasks for patients', and maintaining treatment adherence (15). These responsibilities highlight the prominent role caregivers play in the management of ALS and hence the importance of providing timely support to optimise their well-being (16). Accordingly, understanding how caregivers respond to this potentially unexpected and highly stressful situation is central to improving their well-being and that of the patient. Indeed, examining the coping skills of ALS caregivers may enable clinicians to develop specific caregiver interventions to manage cognitive and behavioural symptoms.

Coping is the psychological process by which people manage stressful, anxiogenic or painful situations. Coping mechanisms have been traditionally divided into task-oriented, emotionoriented and avoidance-oriented styles (17). Task-oriented coping refers to strategies aimed 
1 at directly managing or changing the stressor (i.e., directly targeting the cause of the stress).

2 In contrast, emotion-oriented coping includes strategies designed to regulate emotional

3 reactions to stressors (i.e., focusing on how to respond to the source of stress) (17). Finally,

4 avoidance-oriented coping is defined as strategies undertaken to avoid the source of

5 distress and can be both emotion-focused and task-focused. Alternative taxonomies

6 propose a dysfunctional coping style (18). In general, task-oriented coping strategies tend to

7 be adaptive whilst avoidance is typically associated with worse outcomes such as increased

8 anxiety. Emotion-oriented coping may be highly contextual as it has been linked with both

9 positive and negative outcomes $(18,19)$.

11 Emerging evidence suggests that coping style plays a contributory role in ALS caregiver

12 well-being. Emotion-oriented coping strategies (20), avoidance (21), maladaptive coping strategies such as denial and venting (22) and a passive coping style (23) have all been

14 linked to an increase in burden and distress in ALS caregivers. Caregiver burden and depression are inextricably linked in $\operatorname{ALS}(24,25)$, with depression more common among caregivers compared to patients over time (26). The role of coping in the context of

17 cognitive and behavioural symptoms, however, remains unclear given that studies have

18 typically included samples of patients without overt cognitive and behavioural symptoms.

19 As such, the aim of the present study was to explore the relationships between caregiver 20 coping, psychological morbidity and burden of care in the context of ALS accompanied by 21 additional cognitive and behavioural symptoms. We hypothesised that burden would vary 22 depending on the caregiver coping style and would be greatest among caregivers of patients with cognitive and behavioural symptoms. 


\section{Participants}

3 Fifty-five patients evaluated at specialist tertiary ALS and FTD clinics in Australia were

4 recruited (Neuroscience Research Australia MND Research Clinic/Prince of Wales Hospital

5 MND Clinic, University of Sydney ForeFront ALS/FTD Clinic and the FRONTIER FTD Research

6 Clinic). Patients met current clinical diagnostic criteria for clinically definite or probable ALS

$7(27,28)$ and concomitant probable FTD (29). Diagnosis was established in line with current

8 consensus criteria by a multidisciplinary team comprising of neurologists with expertise in

9 ALS and FTD, psychologists, ALS clinical nurse consultant, and an occupational therapist. All

10 participants underwent comprehensive clinical and neurophysiological investigation and

11 neuropsychological assessment along with structural neuroimaging. Exclusion criteria

12 included severe physical impairment, prior history of other neurological or psychiatric illness

13 that would hinder participation, chronic alcohol or other substance abuse, and limited

14 English proficiency. The primary caregiver was identified by the patient as his/her main

15 informal caregiver (i.e., not paid for their services). Patients without informal caregivers

16 available to complete the caregiver measures were excluded from the study.

18 Ethical approval was granted by the South Eastern Sydney Local Health District [11/103

19 (HREC/11/POWH/148); 10/126] and the University of Sydney Human Research Ethics

20 Committees (2014/050).

Measures

23 Physical status: The revised version of the ALS Functional Rating Scale (ALSFRS-R) (30) is a

24 12-item measure of bulbar, fine motor, gross motor and respiratory functions. Each item is 
1 rated on a four-point scale ranging from 0 (no function) to 4 (normal function) with a total

2 score of 48 indicating normal function.

3

4 Global cognitive function: Global cognitive status was evaluated with the Addenbrooke's

5 Cognitive Examination - III (ACE-III) or its predecessor (ACE-R) $(31,32)$. The ACE-III was

6 administered to patients recruited from 2013 onwards. The ACE-III/ACE-R a brief multi-

7 domain cognitive assessment designed to identify early cognitive symptoms in dementia

8 and comprises five subscales measuring the integrity of the following cognitive abilities:

9 orientation, memory, fluency, language and visuospatial. A total score below 88/100

10 indicated suspected dementia. ACE-III total scores were converted to equivalent ACE-R

11 scores to enable comparison (33).

Behavioural changes: The Motor Neuron Disease Behavioral Scale (MiND-B) (34) is a nine-

14 item informant-completed behavioural measure that evaluates the presence of apathy, disinhibition and stereotypical behaviour. Each item is rated on a scale of 1 (everyday) to 4 (no changes from normal behaviour). A score of $\leq 33$ is indicative of subtle but definite behavioural or executive impairment $(13,34)$ based on the original consensus criteria for the diagnosis of frontotemporal cognitive and behavioural syndromes in ALS (35).

Caregiver psychological morbidity: The short version of the Depression, Anxiety, and Stress Scale-21 (DASS-21) (36) is a self-rated measure of depression, anxiety, and stress symptomatology with seven items tapping each emotional state. Each item is rated on a 4point scale ranging from 0 (does not apply to me at all) to 3 (applies to me very much, or most of the time). Total depression, anxiety, and stress subscores range from 0 to 42 , with 
1 higher scores indicative of greater levels of depression, anxiety or stress. The cut-off scores

2 reflecting different levels of stress, anxiety and depression are: stress (normal=0-10,

3 mild $=11-18$, moderate $=19-26$, severe $=27-34$ and extremely severe $=35-42$ ), anxiety

4 (normal=0-6, mild=7-9, moderate $=10-14$, severe $=15-19$, extremely severe $=20-42$ ) and

5 depression (normal=0-9, mild=10-12, moderate $=13-20$, severe $=21-27$ and extremely

6 severe=28-42).

7

8 Caregiver coping: The Brief COPE (37) was developed as a short version of the COPE

9 Inventory (18). The Brief COPE is a 28-item measure of 14 different coping strategies. Each

10 item is rated on a 4-point scale ranging from 1 (I haven't been doing this at all) to 4 (I've

11 been doing this a lot). Three subscales reflecting dysfunctional, problem-focused and

emotion-focused strategies are derived by summing the relevant component item scores and converting that sum to a percentage of the maximum possible score (Figure 1) $(18,38$ -

14 40).

Caregiver burden: The short version of the Zarit Burden Interview (ZBI) (41) is a 12-item

measure of perceived burden which has been used extensively to study caregiver burden in dementia. Each item is rated on a scale ranging from 0 (Never) to 4 (Nearly Always). Total scores range from 0 to 48 , with higher scores indicative of greater levels of burden. A score of $\geq 17$ is indicative of significant burden in dementia.

\section{Statistical analyses}

Data were screened and analysed using IBM SPSS Statistics for Macintosh, Version 26.0.

24 Descriptive statistics including normality (using the Shapiro-Wilk tests interpreted in 
1 conjunction with histograms, probability-probability plots and the values of skew and

2 kurtosis) were examined to describe the characteristics of the sample and check for any

3 gross violation of the assumptions underlying statistical tests used. A median split on the ZBI

4 was used to classify caregivers with low ( $<13 \mathrm{ZBI}$ total score) and high ( $\geq 13 \mathrm{ZBI}$ total score)

5 burden of care to minimise the probability of type II error due to highly unequal sample

6 sizes based on the cut-off score for dementia (ZBI total scores $\geq 17$ ). Demographic and

7 clinical differences between low and high burden caregivers were analysed by independent

8 sample $t$ tests or Mann-Whitney $U$ tests where appropriate. The relationship between

9 caregiver coping strategies, psychological morbidity and burden were investigated using hierarchical multiple regression analyses, controlling for potential demographic and clinical

11 confounders (to minimise overfitting the regression model). A $p$ value of $<.05$ was considered statistically significant for all tests, with the exception of the Bonferroni corrections applied for multiple comparisons.

\section{RESULTS}

\section{Demographic and clinical characteristics}

The cohort of ALS patients consisted of 39 males and 16 females $(N=55)$ with a mean age of $63.7(S D=10.3)$ years. Approximately $33 \%$ of patients had concomitant FTD. The mean disease duration from symptom onset was 31.0 ( $S D=28.1$ ) months, with a mean ALSFRS-R score of $38.9(S D=7.6)$, indicative of mild-moderate disease. Caregivers had a mean age of $61.9(S D=10.1)$ years and were predominantly patients' spouse $(95 \%)$. The mean ZBI was $13.6(S D=8.1)$, with a minority of caregivers $(31 \%)$ indicating burden levels comparable to those reported in the literature in dementia caregivers (i.e., ZBI total scores $\geq 17$ ). 
1 Basic demographic (sex distribution, age, education level) and disease (disease duration,

2 disease severity) were similar in the two subgroups of ALS patients (Table 1). In contrast,

3 caregivers with high burden (based on median split on the ZBI) were more likely to be caring

4 for a patient diagnosed with ALS-FTD compared to caregivers with lower burden, $\chi^{2}(1, n=55)$

$5=7.2, p<.05$. Cognitive impairment $(U=127.0, z=-2.1, p<.05)$ and behavioural changes

6 extending to disinhibition $(U=167.5, z=-3.4, p<.02)$, apathy $(U=131.0, z=-4.1, p<.02)$ and

7 stereotypical behaviour $(U=181.5, z=-3.3, p<.02)$ were also higher among patients of high-

8 burden caregivers compared to those with low-burden caregivers (lower MiND-B scores

9 reflect greater behavioural changes).

\section{Coping, psychological wellbeing and burden}

12 Overall, caregivers reported using problem-focused strategies more frequently $(M=71.4$, $S D=15.3)$ than emotion-focused $(M=60.8, S D=12.3)$ and dysfunctional $(M=42.2, S D=8.6)$ coping strategies. There were no significant differences in problem-focused $[t(51)=1.4$, $p=.181$, two tailed], emotion-focused $[\mathrm{t}(50)=0.3, p=.750]$ and dysfunctional coping scores

$16[\mathrm{t}(50)=-.6, p=.584$, two-tailed) between low- and high-burden caregivers, after applying a

17 Bonferroni correction. Among the problem-focused strategies, caregivers used planning

$18(M=78.2, S D=17.8)$ more frequently than active coping $(M=75.7, S D=18.4)$ and instrumental support ( $M=65.3, S D=21.0)$. Low- and high-burden caregivers also did not differ on planning $20[\mathrm{t}(52)=-.6, \mathrm{p}=.540$, two-tailed), active $[\mathrm{t}(52)=.7, p=.465$ two-tailed) and instrumental coping scores $[\mathrm{t}(52)=1.4, p=.182$, two-tailed), after applying a Bonferroni correction. 
1 In addition, the majority of caregivers reported normal levels of stress (50\%), anxiety (96\%)

2 and depression (70\%). When present, symptoms of stress (42\%), anxiety (2\%) and

3 depression (20\%) were predominantly in the mild range (Figure 2).

4

5 Hierarchical multiple regression analyses were performed to determine the contribution of

6 coping strategies to caregiver burden after controlling for the presence of cognitive and

7 behavioural deficits. As such, ALS-FTD diagnosis was entered at Step 1 to take into account

8 these deficits without overfitting the regression model. This model was significant and

9 explained $15 \%$ of the variance in caregiver burden. After the entry of emotion-focused, problem-focused and dysfunctional coping strategies at Step 2, the total variance explained by the model as a whole was $31 \%, F(4,47)=5.3, p<.05$. The three coping strategies explained an additional $16 \%$ of the variance in caregiver burden, after controlling for ALSFTD diagnosis, R Squared change $=.16, \mathrm{~F}$ change $(3,47)=3.6, p<.05$. In the final model, only

14 ALS-FTD diagnosis $(\beta=0.6, \mathrm{p}<.05)$ and problem-focused strategies $(\beta=-0.4, p<.05)$ were 15 statistically significant (Table 2 ).

17 Separate regression analyses were carried out to determine the contribution of psychological morbidity to burden. After controlling for ALS-FTD diagnosis, caregiver depression, anxiety and stress were found not be significant predictors of caregiver burden

20 (R Squared change $=.09, \mathrm{~F}$ change $(3,45)=1.9, p<.05)$. Only, ALS-FTD diagnosis $(\beta=0.4$, $\mathrm{p}<.05$ ) was predictive of caregiver burden (Table 3). 
2 Our study demonstrates that the presence of cognitive and behavioural symptoms in

3 patients diagnosed with ALS is a major source of high caregiver burden. Indeed, caregivers

4 of patients with ALS-FTD were three times more likely to report higher burden. In addition, a

5 diagnosis of ALS-FTD, caregiver burden was further predicted by adopting less problem-

6 focused strategies. As such, caregiver support specifically focusing on maximising problem-

7 focused strategies, in addition to information on cognitive and behavioural changes, may be 8 particularly beneficial as part of ALS care.

9

10 In dementia, cognitive and behavioural symptoms are well known variables that are

11 particularly burdensome for caregivers (42). These findings parallel that of the growing research in ALS which shows the negative effects of cognitive and behavioural symptoms on caregiver personal time and health $(10,12,43)$. Notably, apathy, disinhibition and executive

14 impairment have been linked to greater caregiver burden $(9,10,44-47)$, particularly the developmental (e.g. being unable to live their own life) and emotional (e.g., negative

16 feelings towards the patient) aspects of caregiving (11).

As such, support for caregivers of ALS patients presenting with cognitive and behavioural symptoms is often recommended to assist with management of non-motor manifestations of the disease (48). Less is known, however, about the appropriateness and relevance of various types of interventions. Recommended strategies for caregivers of ALS patients with cognitive and behavioural symptoms are based on the general dementia literature. They typically focus on educating and helping caregivers adjust their expectations about patients' reduced capacity to perform day-to-day tasks as well as environmental modifications to 
1 ensure patient's safety (e.g., hiding car keys from patient). Variables specific to ALS,

2 however, present added challenges in implementing caregiver interventions and may have

3 deterred previous attempts to develop interventions (49). Indeed, the rapid physical

4 deterioration associated with ALS is likely to restrict the time and effort caregivers have

5 available to implement specific strategies. In other words, management of

6 cognitive/behavioural symptoms may be considered less pressing compared to increasingly

7 disabling physical symptoms in this patient population. This highlights the importance of

8 developing caregiver support that are specific to this population and can be easily

9 integrated and generalised in ALS care settings.

11 Our results demonstrated that strategies focusing on optimising caregiver's problem-

12 focused strategies may be especially beneficial. Here, caregivers most frequently utilised planning as a coping strategy which involves strategy-thinking about ways to solve a

14 particular problem (37). Indeed, maladaptive coping strategies such as avoidance (21), defensiveness and a reactive coping style (23) as well as denial and venting (22) have been

16 found to be associated with greater caregiver burden and psychological morbidity among

17 ALS caregivers. In the present study, dysfunctional coping strategies, including avoidance, denial, self-blame, and other detrimental ways of coping such as substance abuse were also showed to contribute towards increased caregiver burden, although this did not reach statistical significance.

In FTD, caregiver interventions have been demonstrated to be helpful in reducing psychological morbidity. Provision of a 15-week group intervention to assist FTD caregivers with identifying modifiable and non-modifiable characteristics of stressors (cognitive 
1 appraisal) and developing appropriate coping strategies was associated with reduced

2 caregiver burden and distress associated with behavioural symptoms following the

3 intervention (50). An intervention that focused on providing skills to caregivers to address

4 behavioural changes was also found to benefit this population (51). Similarly, a 5-weekly

5 individual intervention focusing on positive-emotion skill building for family caregivers of

6 FTD patients had a beneficial effect on negative affect, burden, and stress (52). In the

7 context of ALS-FTD, early intervention optimising problem-focused strategies with ALS-FTD

8 caregivers delivered within a multidisciplinary care setting (e.g., initiating care management

9 decisions and advance care planning with the ALS clinical nurse consultant early in the course of the disease) appears a promising avenue to help prevent an increase in caregiver

11 burden (53).

13 Unlike previous reports $(9-11,43,44)$, caregiver depression, anxiety and stress were not 14 predictive of burden of care in the present study. This is perhaps not surprising as symptoms of depression, anxiety and stress reported by caregivers were predominantly within the normal or mild range in the present study. Stress has been shown to contribute to caregiver

17 burden more so than depression and anxiety in ALS (45). Furthermore, recruitment of patients from specialised clinics may have introduced a sample bias whereby severely physically and cognitively and behaviourally impaired patients and their highly burdened caregivers were less likely to attend clinics, therefore underestimating the prevalence and impact of caregiver burden and distress. The low prevalence of ALS-FTD patients may have also resulted in lower rates of burden and psychological morbidity compared to that observed in dementia. 
1 In dementia, evidence suggests that caregivers who are more depressed tend to report

2 greater behavioural and psychological symptoms exhibited by patients (54). Such findings

3 highlight the importance of caregiver variables in their evaluation of "role captivity"

4 (caregiver feelings of being "trapped" in their role) and caregiver overload (e.g., fatigue and

5 burnout) which are major factors underlying caregiver strain in dementia (55). Indeed, a

6 limitation of the present study was that caregiver variables such as use of informal (e.g.,

7 assistance from other family members) and formal supports (e.g., psychological therapy,

8 patient allied health and other therapy) which may have influenced caregiver reports of

9 burden and distress were not examined. Future research incorporating assessment of

10 support utilisation may minimise the potential effects of caregiver variables on ratings of

11 psychological wellbeing and may shed further light on other sociocultural factors

12 contributing to caregiver burden.

14 In conclusion, it is now well established that cognitive and behavioural symptoms are prominent non-motor manifestation of ALS, but there remains a lack of knowledge on

16 specific caregiver support strategies to assist with the management of these symptoms. A

17 focus on active planning delivered within a multidisciplinary care setting offers an avenue

18 for ALS-FTD caregivers to proactively cope with cognitive and behavioural symptoms that

19 will lead to improved care and reduce the risk of caregiver burnout. 
2 This work was supported in part by funding to ForeFront, a large collaborative research

3 group dedicated to the study of frontotemporal dementia and motor neuron disease, from

4 the National Health and Medical Research Council (NHMRC) of Australia program grant

5 (APP1037746) and the Australian Research Council (ARC) Centre of Excellence in Cognition

6 and its Disorders (CE11000102). RA is supported by an NHMRC Early Career Fellowship

7 (GNT1120770). MCK was supported by NHMRC Practitioner Fellowship (1156093), Program

8 Grant (APP1132524) and Partnership Project (APP1153439). OP is supported by an NHMRC

9 Senior Research Fellowship (GNT1103258).

10 
2 The authors report no conflict of interest.

3 
Jashelle Caga Dr Jashelle Caga is a Health Psychologist and Postdoctoral Fellow at the ForeFront MND/ FTD Clinic, Brain \& Mind Centre, University of Sydney.

Margaret C. Zoing Ms Margaret C. Zoing is the ALS Nurse Consultant at the ForeFront MND/ FTD Clinic, Brain \& Mind Centre, University of Sydney.

David Foxe Mr David Foxe is a Clinical Neuropsychologist and Senior Research Officer at the FRONTIER FTD Research Group, Brain \& Mind Centre, University of Sydney.

Eleanor Ramsey Ms Eleanor Ramsey is the Research Coordinator at the ForeFront MND/ FTD Clinic, Brain \& Mind Centre, University of Sydney.

Mirelle D'Mello Ms Mirelle D'Mello is a Psychologist and Research Assistant at the FRONTIER FTD Research Group, Brain \& Mind Centre, University of Sydney.

Eneida Mioshi Professor Eneida Mioshi is an Occupational Therapist and Chair of Dementia Care Research at the School of Health Sciences at the University of East Anglia.

Rebekah M. Ahmed Associate Professor Rebekah Ahmed is a NHMRC Early Career Fellow at the University of Sydney and staff specialist Neurologist at Royal Prince Alfred Hospital, where she directs the multidisciplinary Memory and Cognition Clinic.

Matthew C. Kiernan Professor Matthew C. Kiernan is the Bushell Chair of Neurology at the University of Sydney, Co -Director of the Brain and Mind Centre - Discovery and Translation and staff specialist Neurologist at Royal Prince Alfred Hospital.

Olivier Piguet Professor Olivier Piguet is a NHMRC Senior Research Fellow and Professor of Clinical Neuropsychology at the University of Sydney. He is the director of FRONTIER, the FTD clinical research group at the Brain and Mind Centre. He is Presidentelect of the Australasian Society for the Study of Brain Impairment (ASSBI). 
2 1. Clark CM, Forman MS. Frontotemporal lobar degeneration with motor neuron

3 disease: a clinical and pathological spectrum. Arch Neurol. 2006;63(4):489-90.

42 Kiernan MC, Vucic S, Cheah BC, Turner MR, Eisen A, Hardiman O, et al. Amyotrophic 5 lateral sclerosis. Lancet. 2011;377(9769):942-55.

63 3. Hardiman $\mathrm{O}$, van den Berg LH, Kiernan MC. Clinical diagnosis and management of 7 amyotrophic lateral sclerosis. Nat Rev Neurol. 2011;7(11):639-49.

8 4. Eisen A, Braak H, Del Tredici K, Lemon R, Ludolph AC, Kiernan MC. Cortical influences 9 drive amyotrophic lateral sclerosis. J Neurol Neurosurg Psychiatry. 2017;88(11):917-24.

10 5. Montuschi A, lazzolino B, Calvo A, Moglia C, Lopiano L, Restagno G, et al. Cognitive Neurosurg Psychiatry. 2015;86(2):168-73.

6. Phukan J, Elamin M, Bede P, Jordan N, Gallagher L, Byrne S, et al. The syndrome of cognitive impairment in amyotrophic lateral sclerosis: a population-based study. J Neurol Neurosurg Psychiatry. 2012;83(1):102-8.

7. Burrell JR, Kiernan MC, Vucic S, Hodges JR. Motor neuron dysfunction in frontotemporal dementia. Brain. 2011;134(Pt 9):2582-94.

8. Lomen-Hoerth C, Anderson T, Miller B. The overlap of amyotrophic lateral sclerosis and frontotemporal dementia. Neurology. 2002;59(7):1077-9.

9. Watermeyer TJ, Brown RG, Sidle KC, Oliver DJ, Allen C, Karlsson J, et al. Impact of disease, cognitive and behavioural factors on caregiver outcome in amyotrophic lateral sclerosis. Amyotroph Lateral Scler Frontotemporal Degener. 2015;16(5-6):316-23. 10. Burke T, Elamin M, Galvin M, Hardiman O, Pender N. Caregiver burden in amyotrophic lateral sclerosis: a cross-sectional investigation of predictors. J Neurol. 2015;262(6):1526-32.

11. Chio A, Vignola A, Mastro E, Giudici AD, lazzolino B, Calvo A, et al. Neurobehavioral symptoms in ALS are negatively related to caregivers' burden and quality of life. Eur J Neurol. 2010;17(10):1298-303.

12. Tremolizzo L, Pellegrini A, Susani E, Lunetta C, Woolley SC, Ferrarese C, et al. Behavioural But Not Cognitive Impairment Is a Determinant of Caregiver Burden in Amyotrophic Lateral Sclerosis. Eur Neurol. 2016;75(3-4):191-4.

13. Hsieh S, Caga J, Leslie FV, Shibata M, Daveson N, Foxe D, et al. Cognitive and Behavioral Symptoms in ALSFTD: Detection, Differentiation, and Progression. J Geriatr Psychiatry Neurol. 2016;29(1):3-10.

14. Hogden A, Greenfield D, Nugus P, Kiernan MC. What are the roles of carers in decision-making for amyotrophic lateral sclerosis multidisciplinary care? Patient Prefer Adherence. 2013;7:171-81.

15. Olney RK, Murphy J, Forshew D, Garwood E, Miller BL, Langmore S, et al. The effects of executive and behavioral dysfunction on the course of ALS. Neurology. 2005;65(11):17747.

16. Weisser FB, Bristowe K, Jackson D. Experiences of burden, needs, rewards and resilience in family caregivers of people living with Motor Neurone Disease/Amyotrophic Lateral Sclerosis: A secondary thematic analysis of qualitative interviews. Palliat Med. 2015;29(8):737-45.

17. Lazarus RS, Folkman S. Stress, appraisal, and coping. . New York: Springer; 1984. 
18. Carver CS, Scheier MF, Weintraub JK. Assessing coping strategies: a theoretically based approach. J Pers Soc Psychol. 1989;56(2):267-83.

19. Carver CS, Connor-Smith J. Personality and coping. Annu Rev Psychol. 2010;61:679704.

20. Siciliano M, Santangelo G, Trojsi F, Di Somma C, Patrone M, Femiano C, et al. Coping strategies and psychological distress in caregivers of patients with Amyotrophic Lateral Sclerosis (ALS). Amyotroph Lateral Scler Frontotemporal Degener. 2017;18(5-6):367-77. 21. Rabkin JG, Albert SM, Rowland LP, Mitsumoto H. How common is depression among ALS caregivers? A longitudinal study. Amyotroph Lateral Scler. 2009;10(5-6):448-55.

22. Quattropani MC, La Foresta S, Russo M, Faraone C, Pistorino G, Lenzo V, et al. Emotional burden and coping strategies in amyotrophic lateral sclerosis caregivers: The role of metacognitions. . Minerva Psichiatrica. 2018;59(2):95-104.

23. Creemers $\mathrm{H}$, de Moree $\mathrm{S}$, Veldink JH, Nollet F, van den Berg LH, Beelen A. Factors related to caregiver strain in ALS: a longitudinal study. J Neurol Neurosurg Psychiatry. 2016;87(7):775-81.

24. Chio A, Gauthier A, Calvo A, Ghiglione P, Mutani R. Caregiver burden and patients' perception of being a burden in ALS. Neurology. 2005;64(10):1780-2.

25. Pagnini F, Rossi G, Lunetta C, Banfi P, Castelnuovo G, Corbo M, et al. Burden, depression, and anxiety in caregivers of people with amyotrophic lateral sclerosis. Psychol Health Med. 2010;15(6):685-93.

26. Gauthier A, Vignola A, Calvo A, Cavallo E, Moglia C, Sellitti L, et al. A longitudinal study on quality of life and depression in ALS patient-caregiver couples. Neurology. 2007;68(12):923-6.

27. Brooks BR, Miller RG, Swash M, Munsat TL, World Federation of Neurology Research Group on Motor Neuron D. El Escorial revisited: revised criteria for the diagnosis of amyotrophic lateral sclerosis. Amyotroph Lateral Scler Other Motor Neuron Disord. 2000;1(5):293-9.

28. de Carvalho M, Dengler R, Eisen A, England JD, Kaji R, Kimura J, et al. Electrodiagnostic criteria for diagnosis of ALS. Clin Neurophysiol. 2008;119(3):497-503. 29. Rascovsky K, Hodges JR, Knopman D, Mendez MF, Kramer JH, Neuhaus J, et al. Sensitivity of revised diagnostic criteria for the behavioural variant of frontotemporal dementia. Brain. 2011;134(Pt 9):2456-77.

30. Cedarbaum JM, Stambler N, Malta E, Fuller C, Hilt D, Thurmond B, et al. The ALSFRS$R$ : a revised ALS functional rating scale that incorporates assessments of respiratory function. BDNF ALS Study Group (Phase III). J Neurol Sci. 1999;169(1-2):13-21.

31. Mioshi E, Dawson K, Mitchell J, Arnold R, Hodges JR. The Addenbrooke's Cognitive Examination Revised (ACE-R): a brief cognitive test battery for dementia screening. Int J Geriatr Psychiatry. 2006;21(11):1078-85.

32. Hsieh S, Schubert S, Hoon C, Mioshi E, Hodges JR. Validation of the Addenbrooke's Cognitive Examination III in frontotemporal dementia and Alzheimer's disease. Dement Geriatr Cogn Disord. 2013;36(3-4):242-50.

33. So M, Foxe D, Kumfor F, Murray C, Hsieh S, Savage G, et al. Addenbrooke's Cognitive Examination III: Psychometric Characteristics and Relations to Functional Ability in Dementia. J Int Neuropsychol Soc. 2018;24(8):854-63.

34. Mioshi E, Hsieh S, Caga J, Ramsey E, Chen K, Lillo P, et al. A novel tool to detect behavioural symptoms in ALS. Amyotroph Lateral Scler Frontotemporal Degener. 2014;15(34):298-304. 
35. Strong MJ, Grace GM, Freedman M, Lomen-Hoerth C, Woolley S, Goldstein LH, et al. Consensus criteria for the diagnosis of frontotemporal cognitive and behavioural syndromes in amyotrophic lateral sclerosis. Amyotroph Lateral Scler. 2009;10(3):131-46.

36. Lovibond S, Lovibond P. Manual for the Depression Anxiety Stress Scales. . Sydney: Psychology Foundation; 1995.

37. Carver CS. You want to measure coping but your protocol's too long: consider the brief COPE. Int J Behav Med. 1997;4(1):92-100.

38. Coolidge FL, Segal DL, Hook JN, Stewart S. Personality disorders and coping among anxious older adults. J Anxiety Disord. 2000;14(2):157-72.

39. Cooper C, Katona C, Livingston G. Validity and reliability of the brief COPE in carers of people with dementia: the LASER-AD Study. J Nerv Ment Dis. 2008;196(11):838-43.

40. Cooper C, Katona C, Orrell M, Livingston G. Coping strategies, anxiety and depression in caregivers of people with Alzheimer's disease. Int J Geriatr Psychiatry. 2008;23(9):929-36. 41. Bedard M, Molloy DW, Squire L, Dubois S, Lever JA, O'Donnell M. The Zarit Burden Interview: a new short version and screening version. Gerontologist. 2001;41(5):652-7. 42. Cheng ST. Dementia Caregiver Burden: a Research Update and Critical Analysis. Curr Psychiatry Rep. 2017;19(9):64.

43. Cui B, Cui LY, Liu MS, Li XG, Ma JF, Fang J, et al. Behavioral Symptoms in Motor Neuron Disease and Their Negative Impact on Caregiver Burden. Chin Med J (Engl). 2015;128(17):2295-300.

44. Andrews SC, Pavlis A, Staios M, Fisher F. Which behaviours? Identifying the most common and burdensome behaviour changes in amyotrophic lateral sclerosis. Psychol Health Med. 2017;22(4):483-92.

45. Lillo P, Mioshi E, Hodges JR. Caregiver burden in amyotrophic lateral sclerosis is more dependent on patients' behavioral changes than physical disability: a comparative study. BMC Neurol. 2012;12:156.

46. Caga J, Hsieh S, Highton-Williamson E, Zoing MC, Ramsey E, Devenney E, et al. The burden of apathy for caregivers of patients with amyotrophic lateral sclerosis. Amyotroph Lateral Scler Frontotemporal Degener. 2018:1-7.

47. Caga J, Turner MR, Hsieh S, Ahmed RM, Devenney E, Ramsey E, et al. Apathy is associated with poor prognosis in amyotrophic lateral sclerosis. Eur J Neurol. 2016;23(5):891-7.

48. Miller RG, Jackson CE, Kasarskis EJ, England JD, Forshew D, Johnston W, et al. Practice parameter update: the care of the patient with amyotrophic lateral sclerosis: multidisciplinary care, symptom management, and cognitive/behavioral impairment (an evidence-based review): report of the Quality Standards Subcommittee of the American Academy of Neurology. Neurology. 2009;73(15):1227-33.

49. Merrilees J, Klapper J, Murphy J, Lomen-Hoerth C, Miller BL. Cognitive and behavioral challenges in caring for patients with frontotemporal dementia and amyotrophic lateral sclerosis. Amyotroph Lateral Scler. 2010;11(3):298-302.

50. Mioshi E, McKinnon C, Savage S, O'Connor CM, Hodges JR. Improving burden and coping skills in frontotemporal dementia caregivers: a pilot study. Alzheimer Dis Assoc Disord. 2013;27(1):84-6.

51. O'Connor CMC, Mioshi E, Kaizik C, Fisher A, Hornberger M, Piguet O. Positive behaviour support in frontotemporal dementia: A pilot study. Neuropsychol Rehabil. 2020:1-24. 
1 52. Dowling GA, Merrilees J, Mastick J, Chang VY, Hubbard E, Moskowitz JT. Life 2 enhancing activities for family caregivers of people with frontotemporal dementia.

3 Alzheimer Dis Assoc Disord. 2014;28(2):175-81.

4 53. Hsieh S, Leyton CE, Caga J, Flanagan E, Kaizik C, O'Connor CM, et al. The Evolution of 5 Caregiver Burden in Frontotemporal Dementia with and without Amyotrophic Lateral 6 Sclerosis. J Alzheimers Dis. 2016;49(3):875-85.

7 54. Dunkin JJ, Anderson-Hanley C. Dementia caregiver burden: a review of the literature 8 and guidelines for assessment and intervention. Neurology. 1998;51(1 Suppl 1):S53-60;

9 discussion S5-7.

10 55. Campbell P, Wright J, Oyebode J, Job D, Crome P, Bentham P, et al. Determinants of 11 burden in those who care for someone with dementia. Int J Geriatr Psychiatry.

12 2008;23(10):1078-85. 
Table 1

2 Demographic and clinical characteristics of the study cohort stratified by caregiver burden

3 level

\begin{tabular}{|c|c|c|c|}
\hline & $\begin{array}{l}\text { Low burden } \\
\quad(n=28)\end{array}$ & $\begin{array}{l}\text { High burden } \\
\quad(n=27)\end{array}$ & $p$ value \\
\hline \multicolumn{4}{|l|}{ Patient } \\
\hline Male & $18(33 \%)$ & $21(38 \%)$ & .421 \\
\hline Age (years) & $64.7(9.6)$ & $62.6(11.0)$ & .448 \\
\hline Education (years) & $12.7(3.1)$ & $12.3(3.3)$ & .695 \\
\hline Symptom duration (months) & $21.0(0.9-126.9)$ & $23.0(1.9-138.1)$ & .527 \\
\hline ALSFRS-R total score & $43.0(23.0-47.0)$ & $35.5(22.0-48.0)$ & .108 \\
\hline ALS-FTD diagnosis & $4(22)$ & $14(78)$ & $.007^{*}$ \\
\hline ACE total score & 89.7 (49.1-99.7) & 80.8 (68.9-99.0) & $.035^{*}$ \\
\hline MiND-B Disinhibition score & $16.0(4.0-16.0)$ & $14.0(6.0-16.0)$ & $.001^{* *}$ \\
\hline MiND-B Apathy score & $10.0(4.0-12.0)$ & $6.0(3.0-12.0)$ & $.0001 * *$ \\
\hline MiND-B Stereotypical score & $8.0(3.0-8.0)$ & $5.0(2.0-8.0)$ & $.001 * *$ \\
\hline \multicolumn{4}{|l|}{ Caregiver } \\
\hline Male & $10(18 \%)$ & $15(27 \%)$ & .228 \\
\hline Age (years) & $60.9(9.0)$ & $63.0(11.2)$ & .437 \\
\hline Education (years) & $11.0(9.0-17.5)$ & $13.0(6.0-18.0)$ & .139 \\
\hline DASS-21 Depression score & $4.0(0.0-14.0)$ & $8.0(0.0-38.0)$ & .107 \\
\hline DASS-21 Anxiety score & $0.0(0.0-12.0)$ & $2.0(0.0-8.0)$ & .104 \\
\hline DASS-21 Stress score & $6.0(0.0-32.0)$ & $12.0(2.0-42.0)$ & .060 \\
\hline
\end{tabular}

Abbreviations: $A C E=$ Addenbrooke's Cognitive Examination; ALSFRS-R = ALS Functional

Rating Scale- Revised; ALS-FTD = amyotrophic lateral sclerosis-frontotemporal dementia;

DASS-21= Depression, Anxiety, and Stress Scale-21; MiND-B = Motor Neuron Disease

Behavioural Scale.

Data represent the mean (SD), median (minimum-maximum), or $\mathrm{n}(\%)$.

${ }^{*} p<.05$

$* * p<.02$ (Bonferroni correction)

11

12

13

14 


\section{$1 \quad$ Table 2}

2 Hierarchical multiple regression analysis predicting caregiver burden from coping style

\begin{tabular}{lcccc}
\hline & $\boldsymbol{B}$ & SE B & $\boldsymbol{\beta}$ & $\boldsymbol{p}$ \\
\hline Step 1 & & & & \\
$\quad$ Constant & 11.4 & 1.3 & & .0001 \\
$\quad$ ALS-FTD diagnosis & 6.7 & 2.2 & 0.4 & $.004^{*}$ \\
& & & & \\
Step 2 & 7.3 & 7.1 & & .309 \\
$\quad$ Constant & 8.6 & 2.2 & 0.5 & $.0001^{*}$ \\
ALS-FTD diagnosis & 0.1 & 0.1 & 0.2 & .244 \\
$\quad$ Emotion-focused strategies & -0.2 & 0.1 & -0.4 & $.024^{*}$ \\
$\quad$ Problem-focused strategies & 0.2 & 0.1 & 0.2 & .060 \\
$\quad$ Dysfunctional coping strategies & & & & \\
\hline
\end{tabular}

3 Abbreviations: ALS-FTD = amyotrophic lateral sclerosis-frontotemporal dementia $4 \quad * p<.05$ 


\section{$1 \quad$ Table 3}

2 Hierarchical multiple regression analysis predicting caregiver burden from psychological

3 morbidity

\begin{tabular}{lllll}
\hline & $B$ & $S E B$ & $\beta$ & $p$ \\
\hline
\end{tabular}

\section{Step 1}

Constant

$\begin{array}{cccc}11.4 & 1.3 & .0001 \\ 6.7 & 2.3 & 0.4 & .005^{*}\end{array}$

ALS-FTD diagnosis

\section{Step 2}

Constant

$8.2 \quad 1.9 \quad .0001$

ALS-FTD diagnosis

$\begin{array}{llll}6.5 & 2.3 & 0.4 & .007^{*}\end{array}$

Caregiver depression

$\begin{array}{llll}0.1 & 0.2 & 0.1 & .703\end{array}$

Caregiver anxiety

$\begin{array}{llll}-0.1 & 0.6 & -0.02 & .920\end{array}$

Caregiver stress

$\begin{array}{llll}0.3 & 0.2 & 0.3 & .247\end{array}$

4 Abbreviations: ALS-FTD = amyotrophic lateral sclerosis-frontotemporal dementia

$5 * p<.05$ 


\section{Figure 1}

Title: The Brief COPE (Carver et al., 1997)

Legend: The Brief COPE items divided into three subscales: emotion-focused, problemfocused, and dysfunctional coping strategies 


\section{Figure 2}

Title: ALS caregiver psychological morbidity

Legend: Proportion of ALS caregivers reporting different levels of depression, anxiety and stress. 